\title{
Epidemiological study of bacterial meningitis in Tunisian children, beyond neonatal age, using molecular methods: 2014-2017
}

\author{
Sondes Haddad-Boubaker ${ }^{1,2,3}$, Marwa Lakhal ${ }^{1}$, Cyrine Fathallah ${ }^{1}$, Samar Mhimdi ${ }^{1}$, \\ Aida Bouafsoun ${ }^{1}$, Amel Kechrid ${ }^{1}$, Hanen Smaoui ${ }^{1}$
}

1. Laboratory of Microbiology of Bechir Hamza Children's Hospital, LR18ES39, Faculty of Medicine of Tunis, Tunis El-Manar University, Tunis, Tunisia.

2. Laboratory of Clinical Virology, WHO EMRO Regional Reference Laboratory for Poliomyelitis and Measles, Pasteur Institute of Tunis, Tunisia.

3. Research Laboratory of Virus, Vector and Host, Pasteur Institute of Tunis, Tunis El-Manar University, Tunisia.

\begin{abstract} (Hib). Only Hib vaccination was available during our study period. January 2014 to June 2017. ed by bacterial meningitis.

Keywords: Bacterial meningitis; children; Tunisia; PCR.

DOI: https://dx.doi.org/10.4314/ahs.v20i3.14 dx.doi.org/ 10.4314/abs.v20i3.14

\section{Introduction}

Bacterial meningitis is a devastating disease associated with a high mortality rate and significant disability in survivors over the world ${ }^{1-3}$. Clinical diagnosis is difficult to assess, among infants. Moreover, bacteriological conventional methods present a limited sensitivity for low-quality cerebrospinal fluid (CSF) samples and antibiotic pre-treated cases $^{3,4}$. Thus, bacterial meningitis cases remain underestimated, highlighting the need to use methods with high-performance detection such as real time $\mathrm{PCR}^{4}$.
\end{abstract}

Background: Since the 1990s, the epidemiology of bacterial meningitis worldwide has changed thanks to vaccination. In Tunisia, the main causative pathogens were Streptococcus pneumoniae, Neisseria meningitidis and Haemophilus influenzae serotype b

Objectives: We performed a laboratory case report based-study of suspected bacterial meningitis in Northern Tunisia from

Methods: CSF samples obtained from children beyond neonatal age with suspicion of meningitis were tested by two real time PCRs, targeting pneumococcus, meningococcus and Hib, and conventional methods.

Results: Using real-time PCR, 63 were positive including ten supplementary cases compared to conventional methods. A general decrease of bacterial meningitis cases was demonstrated comparing to previous data. Pneumococcus was predominant (69.84\%) followed by meningococcus (28.57\%) and $\mathrm{Hib}(1.59 \%)$. The main serotypes were 14, 19F, 6B and 23F for pneumococcus and serogroup B for meningococcus. Most cases occurred during cold season and children under one year were the most affect-

Conclusion: Our study suggests the predominance of pneumococcal cases. It may provide valuable data on meningitis epidemiology before the introduction of pneumococcal vaccine, which may be useful for future evaluation.

Cite as: Haddad-Boubaker S, Lakbal M, Fathallah C, Mbimdi S, Bonafsoun A, Kechrid A, et al. Epidemiological study of bacterial meningitis in Tunisian children, beyond neonatal age, using molecular methods: 2014-2017. Afri Health Sci.. 2020;20(3): 1124-1132. bttps://

\section{Corresponding author:}

Marwa Lakhal,

Children's Hospital Bechir Hamza,

Laboratory of Microbiology

Email: maroua.lakhal@gmail.com
Children beyond neonatal age are among the most exposed to bacterial meningitis. Historically, the major causative pathogens for this population worldwide were Neisseria meningitidis (N. meningitidis), Streptococcus pneumoniae (S. pneumoniae) and Haemopbilus influenzae serotype b (Hib) ${ }^{1}$. However, in the last two decades, bacterial meningitis epidemiology changed thanks to the introduction of conjugate vaccines against the three bacteria. Indeed, the vaccination against Hib reduced the number of Hib meningitis cases by $90 \%$ in developed countries $^{5}$. Additionally, the successive introduction of pneumococcal conjugate vaccines (PCV7, PCV10 and PCV13) reduced the number of meningitis cases and limited the possible serotypes replacement ${ }^{5,6}$. Furthermore, the conjugate vaccine against $N$. meningitidis serogroup $C$ lowered the number of invasive diseases caused by this bacterium in different region of the world. For instance, in the United Kingdom, where meningococcus 
serogroup C conjugate vaccine was introduced in 1999 , the number meningococcal invasive diseases decreased by $81 \%$, from 1998 to 2001, with no evidence of serogroup replacement during the two years following the vaccine introduction ${ }^{5}$.

In Tunisia, a middle-income country, these three pathogens were the major agents of bacterial meningitis in children $^{7,8}$. Most frequent serotypes were 14 and $23 \mathrm{~F}$ for $S$. pneumoniae, serotype $\mathrm{b}$ for $H$. influenzae and serogroup B for $N$. meningitidis ${ }^{8,9}$. Hib vaccination was introduced during the 2002-2005 period, in the national vaccination schedule, leading to the decrease of Hib meningitis incidence ${ }^{8,10}$. However, the use of Hib vaccine was interrupted from 2005 to 2011 causing a rise of Hib meningitis cases ${ }^{8,11}$. Since 2011, it has been reintroduced a second time as a routine vaccination. Furthermore, since April 2019, pneumococcal vaccine has been introduced in the national schedule, using PCV10 ${ }^{12}$. However, little data about bacterial meningitis epidemiology have been available since $2011^{11}$. Most studies focused on characterisation of one pathogen in a very restricted area ${ }^{13-15}$ and did not consider the impact of the Hib vaccination reintroduction ${ }^{11}$ nor the added value of molecular diagnosis in assessing bacterial meningitis epidemiology.

With the aim of estimating the pathogen distribution of meningitis cases in infants and children in Northern Tunisia, we assessed a laboratory case report basedstudy over a three and half-year period using molecular and conventional methods.

\section{Methods}

\section{Ethics statement and informed consent}

The Ethical Committee of Bechir Hamza Children's Hospital in Tunis, Tunisia, approved this study (number 002/2017). The analysis was performed on the leftover of CSF samples obtained for diagnostic purposes. All information related to patient's identity from the sampling of this study (excluding age and sex) was treated confidentially in accordance with ethical guidelines and the Tunisian legal requirements.

\section{Samples and Study design}

From January 2014 to June 2017, we performed a prospective laboratory case report based-study. All CSF samples received in the laboratory of Microbiology of Bechir Hamza Children's Hospital, for bacterial meningitis diagnosis, were investigated. This hospital is the main paediatric hospital (a bed capacity of 347 and at least 30.625 admissions in 2017) gathering patients from all Northern regions of Tunisia (11/24 governorates), the most populated area, and also referred patients from other regional hospitals. The investigated samples $(n=253)$ were obtained from children aged from 1 month to 15 years old, presenting various meningitis signs such as high fever, neck stiffness, photophobia, hypotonia and seizures ${ }^{3,11}$.

CSF samples were evaluated by bacteriological conventional methods, i.e. bacterial culture, direct examination for microscopic detection of bacteria and Gram staining, and latex agglutination test ${ }^{16}$. Samples, characterised by at least 5 leukocytes per $\mathrm{mm}^{3}$, were selected and considered as suspected bacterial meningitis cases. These CSF samples were considered for PCR when they had a sufficient volume, i.e. at least $200 \mu$ L. They were stored at $-20^{\circ} \mathrm{C}$ until molecular investigations..

The reference strain of Streptococcus pneumoniae (ATCC 49619) and field strains of Neisseria meningitidis and Hib were used as positive controls for real time PCR tests. Field strains were obtained from CSF for H. influenzae serotype $\mathrm{b}$ meningitis case and blood culture for meningococcaemia case and isolated in the laboratory of Microbiology of Bechir Hamza Children's Hospital in Tunis (Tunisia).

\section{Molecular diagnosis of bacterial meningitis}

Bacterial DNA was extracted by heat-shock protocol for positive controls and by using the QIAamp DNA Mini Kit (Qiagen, Hilden, Germany) for CSF samples according to the manufacturer's instructions.

For detection of bacterial meningitis, we performed a multiplex real time PCR for $S$. pneumoniae and N. meningitidis detection and a singleplex real time PCR for Hib detection by using TaqMan chemistry ${ }^{17}$.

\section{Typing methods}

Meningococcal serogroups were identified by latex agglutination test (Pastorex meningitis, BIO-RAD, Marne-la-Coquette, France) and confirmed by PCR ${ }^{18}$. Pneumococcal serotyping was performed by agglutination test (Pneumotest Latex, Statens Serum Institut Diagnostica, Hillerod, Denmark) for strains collected from 2014 to November 2015. Due to the lack of latex agglutination test, several conventional PCRs were performed for strains collected from December 2015 to October 2016 according to the Centers of Disease Control and Prevention (CDC) and World Health Or- 
ganization (WHO) recommendations ${ }^{16}$ as well as Pai et al. ${ }^{19}$, using the Applied Biosystems kit in GeneAmp PCR System 9700 instrument (Applied Biosystems, Foster City, USA) followed by agarose gel electrophoresis.

\section{Statistical analysis}

Statistical significance was determined using EpiInfo software 7.2. The Fisher exact test was performed to compare meningococcal detection proportions by molecular and conventional methods (95\% confidence intervals).

\section{Results}

Detection rates of bacterial meningitis cases

Overall, 4892 CSF samples were obtained from children admitted in the Bechir Hamza Children's Hospital. Based on inclusion criteria, only 253 cases were considered as suspected bacterial meningitis cases, while the others corresponded to viral meningitis cases (4639 cases). All CSF samples ( $\mathrm{n}=253$ ) were investigated by conventional methods and 183 fulfilled the inclusion criteria for PCR testing (sufficient volume of at least $200 \mu \mathrm{L}$ ) and were investigated by real time PCR (Table 1). Among the 183 cases, 31 CSF samples were collected after antibiotic treatment.

Table 1 Yearly distribution of suspected bacterial meningitis tested among children

\begin{tabular}{l|r}
\hline Year & Number of cases \\
\hline 2014 & 87 \\
2015 & 69 \\
2016 & 81 \\
$2017^{\mathrm{a}}$ & 16 \\
Total & 253 \\
${ }^{\mathrm{a}}$ Period between January and June
\end{tabular}

Using real time PCR, 63 cases were detected positive among the 183 investigated cases while using conventional methods, only 53 cases out of 253 had positive results. All positive cases were confirmed by real-time PCR. Furthermore, ten additional positive cases were detected. The 63 positive cases included 44 cases $(69.84 \%)$ of pneumococcal meningitis followed by 18 cases $(28.57 \%)$ of meningococcal meningitis and one case $(1.59 \%)$ of Hib meningitis. The supplementary detected cases corresponded to six meningococcal meningitis and four pneumococcal meningitis cases. Real-time PCR had a significantly higher rate of detection of meningococcal meningitis than conventional methods given that it revealed one-third more positive cases $(\mathrm{P}=0.005)$. Among the $31 \mathrm{CSF}$ samples collected after antibiotic treatment, eleven $(35.5 \%)$ were positive: eight were positive by real time PCR and three were detected by both methods.

\section{Detection rates according to sex and age}

For bacterial meningitis cases, approximately two-thirds corresponded to boys (68.25\%).

Age information was available for 156 suspected meningitis cases: 49 of them were positive cases whereas 107 of them were negative. Among the positive cases, the average age was 2 years and 4 months $(\mathrm{SD}=2$ years and 2 months, IQR $=3$ years and 6.5 months, range $=2$ months-11 years) and the median age was 10 months. Most positive cases corresponded to children aged one year and younger (57.14\%). For children aged between 1 and 2 years, the rate was lower $(8.16 \%)$. For children aged between 3 and 4 years, the rate was $18.37 \%$ (Table $2)$. For the 107 negative cases, most children were aged one year and younger $(63.55 \%$ ) (Table 2). 
Table 2 Distribution of negative and positive cases regarding the age

\begin{tabular}{c|ccccc}
\hline Age (yrs.) & Negative cases & Positive cases & S. pneumoniae & N. meningitidis & H. influenzae b \\
\hline 1 mo-1 & 68 & 28 & 22 & 6 & 0 \\
$>1-2$ & 7 & 4 & 4 & 0 & 0 \\
$>2-3$ & 2 & 0 & 0 & 0 & 0 \\
$>3-4$ & 5 & 9 & 7 & 1 & 1 \\
$>4-5$ & 5 & 0 & 0 & 0 & 0 \\
$>5-11$ & 20 & 8 & 5 & 3 & 0 \\
Total cases & 107 & 49 & 38 & 10 & 1
\end{tabular}

Distribution of meningitis cases according to years and seasons

Pneumococcal meningitis cases were the most observed during our study period; six cases in 2014 and eighteen cases each in 2015 and 2016. For meningococcal meningitis, the number varied from three cases (2015) to six and seven cases (2016 and 2014 respectively). Only one case of Hib was detected in 2015 (Fig. 1).

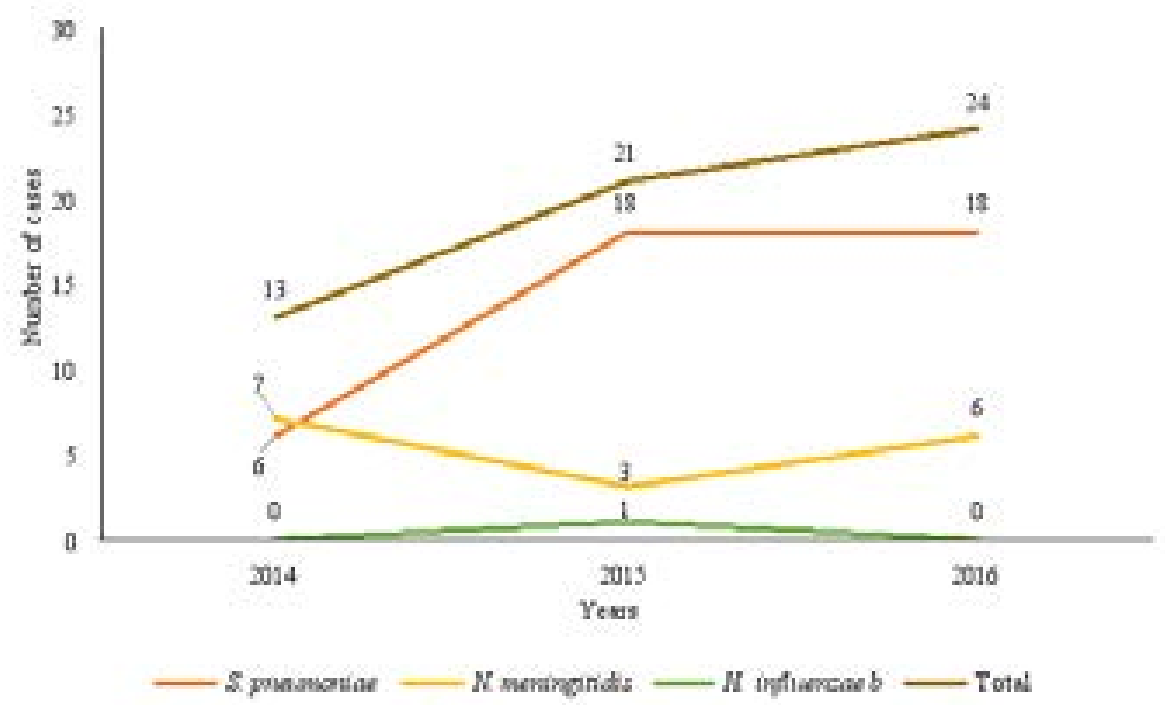

Fig. 1 Number of positive cases of S. pneumoniae, N. meningitidis and $H$. influenzae $b$ according to years.

The distribution of bacterial meningitis cases revealed that most cases had occurred in winter and spring, with
28 cases $(44.44 \%)$ and 18 cases $(28.57 \%)$ out of 63 respectively. (Fig. 2). 


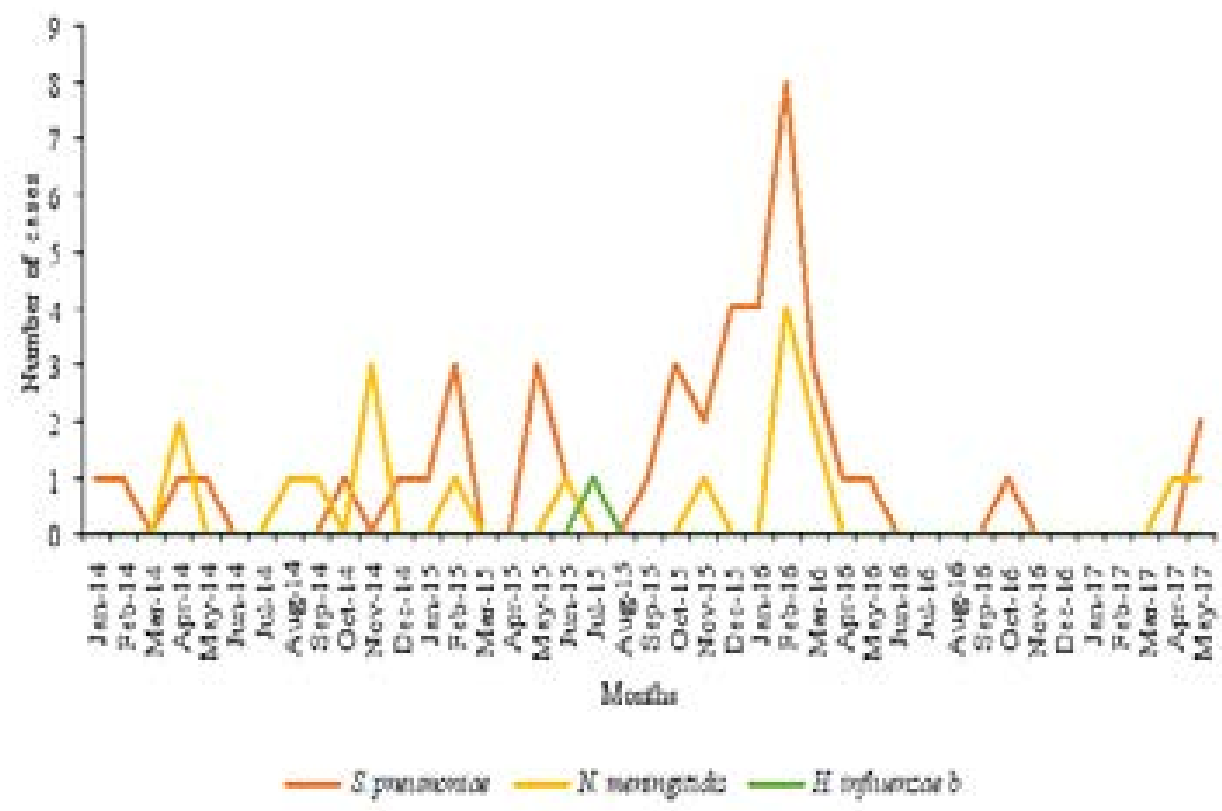

Fig. 2 Monthly distribution of positive bacterial meningitis cases (2014-2017) according to bacterial species.

For pneumococcal meningitis, 23 cases $(52.27 \%)$ occurred in winter, twelve cases $(27.27 \%)$ in the spring, eight cases $(18.18 \%)$ during autumn seasons and only one case $(2.27 \%)$ occurred in summer 2015. Moreover, we have noted a peak of pneumococcal meningitis (27 cases out of 44 cases) from September 2015 to May 2016.

For meningococcal meningitis, six cases $(33.33 \%)$ occurred in winter, six cases $(33.33 \%)$ in spring, two cases $(11.11 \%)$ in summer and four cases $(22.22 \%)$ occurred in autumn 2014.

For $H$. influenzae b meningitis, only one case occurred in July 2015.

\section{Typing}

Among the 18 meningococcal meningitis cases, 12 were positive by conventional methods: ten by culture and latex agglutination and two by direct examination. All the ten culture/latex agglutination positive samples were $N$. meningitidis serogroup B.

Moreover, 34 pneumococcal strains among the 44 positive cases were obtained and typed. Most pneumococcal cases were serotype 14 ( 9 cases) followed by serotypes 19F (8 cases), 6B (4 cases), 23F (4 cases), 6A/B (2 cases), 18 ( 2 cases), 19A ( 2 case), 6A (1 case), 12F (1 case) and $9 \mathrm{~V}$ (1 case). Fluctuations of different serotypes isolates were observed. The peak of pneumococcal meningitis recorded from September 2015 to May 2016 was the consequence of a slight rise of different serotypes, mainly serotype 14 (7 cases) followed by serotype 19F (5 cases) and serotype $23 \mathrm{~F}$ (4 cases).

\section{Discussion}

Bacterial meningitis is a major concern for public health worldwide and especially in developing countries ${ }^{5,16,20,21}$. This study describes the distribution of the three main causative bacteria of meningitis cases in Northern Tunisian children beyond neonates, after the reintroduction of Hib vaccination, and before the introduction of pneumococcal vaccination, from January 2014 to June 2017. It presents valuable results, which can be enlightening on bacterial meningitis in Tunisia given that it concerns the most populated governorates in Tunisia (5.347.531 inhabitants among 11.007.326 recorded in the Tunisian 2014 census by the National Institute of Statistics) ${ }^{22}$.

This study suggested an important decrease or absence of Hib cases in Northern Tunisia (only one case detected in our study) and the predominance of pneumococcal cases. Such epidemiological study should be followed by others studies from Central and Southern Tunisia targeting the majority of health centres to confirm this conclusion. Our results are informative at the national stage and may contribute to reshaping the general view on national vaccination strategy by adapting the selection of pneumococcal vaccine to the circulating serotypes. At the regional level, it may be also of great interest to other low- and middle-income countries experiencing the same situation such as Egypt, Malaysia or $\operatorname{Iran}^{23,24}$.

On the other hand, the added value of this study was the use of real time PCR, which allowed the detection 
of $10 / 62$ additional cases to those obtained by conventional methods, especially for meningococcal cases $(\mathrm{P}=0.005)$. Indeed, it was previously demonstrated that molecular methods, targeting microbial genome, have the advantage to detect unviable bacterium. Therefore, they could overcome $N$. meningitidis alteration during transportation at ambient temperature and detect more positive cases ${ }^{17,25}$. Our significant results highlighted the need for an optimum framework to preserve meningococcus viability especially rapid transportation at $37^{\circ} \mathrm{C}^{25}$.

Our findings demonstrated that bacterial meningitis is still causing damage in children beyond neonates in Northern Tunisia. However, our results revealed a general decrease in the number of bacterial meningitis cases during the study period in comparison with the number of cases obtained in the same region between 2000 and $2011^{8}$. Indeed, Smaoui et al. study (2012) reported 24 to 53 meningitis cases ${ }^{8}$ per year, whereas in our study, we found an average of 16 bacterial meningitis cases per year (13 to 24 cases per year between 2014 and 2016) despite using sensitive detection methods. This result may be explained, first, by the depletion of Hib meningitis cases thanks to the reintroduction of Hib vaccination in 2011: ninety-nine cases were recorded between 2000 and $2011^{8}$ and one case was recorded from 2014 to mid-2017. Hib vaccination was introduced in 2002 and interrupted from 2005 to 2011 leading to the increase of Hib meningitis cases ${ }^{8,11}$. Our findings might suggest the efficiency of the reintroduction of Hib conjugate vaccine since 2011, as previously demonstrated in other regions of the world. Indeed, in high income countries, Hib meningitis cases decreased by $90 \%$ after introduction of Hib vaccination and in the middle- and low-income countries such as Morocco, they decreased by $75 \%{ }^{5,26}$. Nowadays, this pathogen is considered to be eradicated in certain regions such as Europe $^{3}$. Nevertheless, the recorded case corresponded to a vaccinated patient. This patient was given all Hib vaccine doses and thus immunological investigation was recommended. Other reports described Hib meningitis cases after vaccination and explained that it might be due to immunodeficiency or non-respect of vaccination schedule such as lack of booster shots ${ }^{27}$.

Second, we also noted a global decrease of meningococcal cases. For instance, between 2000 and 2011, meningococcal cases varied from 4 to 21 per year ${ }^{8}$ while we recorded 3 cases to 7 cases per year between 2014 and 2016. The high frequency recorded between 2000 and 2011 was mostly related to an outbreak that oc- curred in 2004-2005 (about 35 cases) ${ }^{14,28}$. Thus, continuous surveillance of meningococcal meningitis should be carried out. In other regions of the world such as sub-Saharan Africa and India, after the introduction of Hib and pneumococcal conjugate vaccines, N. meningitidis became a major cause of bacterial meningitis ${ }^{5}$ and caused seasonal outbreaks with significant mortality. Also, in European countries, it became the leading cause of bacterial meningitis ${ }^{3,29-31}$. Several meningococcal conjugate vaccines have been introduced in different regions of the world, such as conjugate meningococcal serogroup $C$ and protein-based serogroup $B$ vaccines in Europe since 1999 and 2015 respectively, and meningococcal serogroup A conjugate vaccine in sub-Saharan Africa since 2010. Many reports proved their effectiveness in the following years, especially meningococcal C and $A$ vaccines, by the significant decrease of meningococcal meningitis cases caused by these serogroups ${ }^{32,33}$. In contrast, the number of pneumococcal meningitis cases remains the same. Indeed, during the 2000-2011 period, an average of 13 cases per year (157 total cases) was reported and in our study period, we found an average of 14 cases (44 total cases). However, the main cases (27/44 cases) were obtained between September 2015 and May 2016. The most detected serotypes were 14, 19F, 23F, 6B and 19A. These fluctuations may describe the natural epidemiological evolution of these serotypes which are endemic within the population, be- cause of a possible weakening of the herd immunity. Nevertheless, we could not rule out the possible occur- rence of outbreaks, especially for serotype 14 . More precise characterization methods were needed to confirm this hypothesis. Additionally, the unchanged number of pneumococcal cases highlighted the need to introduce pneumococcal conjugate vaccines in the Tunisian immunization schedule as recommended by WHO in 200734. Indeed, in April 2019, pneumococcal conjugate vaccine was introduced in the Tunisian vaccination program using PCV10 with three doses at two, four and eleven months ${ }^{12}$. This introduction might lead to a change in bacterial meningitis epidemiology in Tunisia.

The same issue was noticed in other countries ${ }^{35}$. For example, in Morocco, the introduction of pneumococcal conjugate vaccine, since 2010, has reduced the incidence of pneumococcal meningitis in the under-five years from 34.6 cases (2007-2010) to 13.5 cases (2011-2014) per 100000 inhabitants $^{36}$. Same results were shown in Western Europe countries following the introduction of PCV7 then later with PCV10 and or PCV13 ${ }^{37}$. In addition to the depletion of meningitis cases, the in- 
troduction of pneumococcal vaccines may improve the childhood health status by preventing severe respiratory infection, especially for preterm infant. We think that it will be important to maintain this vaccination with high coverage and to avoid interruption, as previously experimented with Hib vaccination in Tunisia. For instance, in Kuwait, despite the introduction of PCV13 vaccine in 2010, S. pneumoniae remained the major cause of bacterial meningitis in children ${ }^{38}$. Sadeq et al study explained that it might be due to the low vaccine coverage, especially for infants less than six months and to a possible non-match of the common serotypes in Kuwait with coverage of PCV13 vaccine serotypes ${ }^{38}$. Thus, serotyping investigations should provide valuable information that enables to adjust vaccination strategies to the epidemiological context ${ }^{14}$. Otherwise, the use of these conjugate vaccines may give rise to the emergence of other uncovered serotypes, such as 6A, 19A and 3 when using PCV10 and 24F, 22F, 8 and 15A if using PCV13/PCV10 ${ }^{37}$.

Even though our study was restricted to the Northern regions of Tunisia, it presented valuable and different results from previous studies in the area ${ }^{8,10,11}$. In this study, we did not include neonatal meningitis cases because the epidemiology is different from children beyond neonatal age since other main pathogens, such as Escherichia coli, Listeria monocytogenes and Streptococcus agalactiae, are implicated. Furthermore, results obtained in our laboratory during the study period showed that S. pneumoniae, N. meningitis and $H$. influenrae serotype b were not detected among neonatal cases (unpublished data). Also, a depth analysis of $6 \mathrm{~A} / 6 \mathrm{~B}$ pneumococcal strains detected by PCR should be carried for precise serotype identification.

In conclusion, this study suggested a depletion or absence of Hib meningitis cases and the predominance of pneumococcal cases in Northern Tunisia, between 2014 and mid-2017. This finding gave an idea of a possible efficiency of Hib vaccination reintroduction, which should be confirmed by other studies targeting other regions of the country, and might be instructive at the local and regional level. On the other hand, it provided valuable data on meningitis epidemiology before the introduction of pneumococcal vaccine, which will be important to evaluate the effectiveness and the serotype coverage of the recently introduced pneumococcal vaccine PCV10.

\section{Conflict of interest}

None declared.

\section{References}

1. Kasanmoentalib ES, Brouwer MC, van der Beek D. Update on bacterial meningitis: epidemiology, trials and genetic association studies. Curr Opin Neurol. 2013; 26:282-8. https://doi.org/10.1097/ WCO.0b013e328360415c

2. Tebruegge M, Curtis N. Epidemiology, etiology, pathogenesis and diagnosis of recurrent bacterial meningitis. Clin Microbiol Rev. 2008;21(3):519-37. https:// doi.org/10.1128/CMR.00009-08

3. van der Beek D, Cabellos C, Dzupova O, Esposito S, Klein M, Kloek AT et al. ESCMID guideline: diagnosis and treatment of acute bacterial meningitis. Clin Microbiol Infect. 2016;22(Suppl 3):37-62. https:/ / doi. org/10.1016/j.cmi.2016.01.007

4. Nhantumbo AA, Cantarelli VV, Caireão J, Munguambe AM, Comé CE, Pinto $\mathrm{G}$ do $\mathrm{C}$ et al. Frequency of Pathogenic Paediatric Bacterial Meningitis in Mozambique: The Critical Role of Multiplex Real-Time Polymerase Chain Reaction to Estimate the Burden of Disease. PLoS One. 2015;10(9): e0138249. https://doi. org/10.1371/journal.pone.0138249

5. Brouwer MC, Tunkel AR, van der Beek D. Epidemiology, diagnosis and antimicrobial treatment of acute bacterial meningitis. Clin Microbiol Rev. 2010; 23:467-92. https://doi.org/10.1128/CMR.00070-09

6. Whitney GC. Changing epidemiology of pneumococcal disease in the era of conjugate vaccines. Curr Epidemiol Rep. 2016; 3:125-35. https://doi.org/10.1007/ s40471-016-0077-5

7. Mezghani Maalej S, Kassis M, Mahjoubi Rhimi F, Damak, J, Hammami A. Bactériologie des méningites communautaires dans la région de Sfax, Tunisie (1993-2001). Med Mal Infect. 2006; 36:105-10. https:// doi.org/10.1016/j.medmal.2005.10.011

8. Smaoui H, Bouafsoun A, Kechrid A. Epidemiology of bacterial meningitis in Tunisian children (2000-2011) [abstract]. Arch Dis Child. 2012;97(Suppl 2):249. https://doi.org/10.1136/archdischild-2012-302724.0867

9. Borrow R, Caugant DA, Ceyhan M, Christensen H, Dinleyici EC, Findlow J et al. Meningococcal disease in the Middle East and Africa: Findings and updates from the Global Meningococcal Initiative. I Infect. 2017; S0163-4453(17):30114-7. https://doi.org/10.1016/j. jinf.2017.04.007

10. Bel Hadj Khelifa A, Mastouri M, Ben Abdallah H, Noomen S, Kheder M. Les méningites purulentes dans la région de Monastir, Tunisie (1999-2006) : aspects bactériologiques et état de résistance aux antibiotiques. Bull Soc Pathol Exot. 2011;104(1):42-8. https:// doi.org/10.1007/s13149-010-0077-5 
11. Sfaihi L, Kamoun F, Kamoun T, Aloulou H, Mezghani S, Hammemi A et al. Les méningites purulentes de l'enfant : aspects épidémiologiques et évolutifs. Tunis Med. $2014 ; 92(2)$ : 141-6.

12. Basic Health Care Direction. Ministry of Public Health of Tunisia. Vaccination calendar April 2019. Available at <http://www.santetunisie.rns.tn/images/ docs/anis/actualite/2019/Mars/calendrier-de-vaccination-avril-2019F.pdf>; 2019. Accessed 13 $3^{\text {th }}$ July 2019.

13. Marzouk M, Ferjani A, Bouafia N, Harb H, Ben Salem Y, Boukadida J. Serotype distribution and antimicrobial resistance of invasive and non-invasive pneumococcal isolates in Tunisia. Microb Drug Resist. 2015;21(1):85-9. https://doi.org/10.1089/ mdr.2014.0084

14. Saguer A, Smaoui H, Taha MK, Kechrid A. Characterization of invasive Neisseria meningitidis strains isolated at the Children's Hospital of Tunis, Tunisia. East Mediterr Health J. 2016;22(5):343-9. https:// doi.org/10.26719/2016.22.5.343

15. Charfi F, Smaoui H, Kechrid A. Non-susceptibility trends and serotype coverage by conjugate pneumococcal vaccines in a Tunisian paediatric population: A 10-year study. Vaccine. 2012;30(Suppl6): G18-24. https://doi.org/10.1016/j.vaccine.2012.07.017

16. Centers of Disease Control and Prevention World Health Organization. Laboratory methods for the diagnosis of meningitis caused by Neisseria meningitidis, Streptococcus pneumoniae and Haemophilus influenzae. Available at $<\mathrm{http}: / /$ apps.who.int/iris/bitstream/handle/10665/70765/WHO_IVB_11.09_eng. pdf? sequence $=1$ \&isAllowed $=y>$; 2011. Accessed $17^{\text {th }}$ November 2015.

17. Haddad-Boubaker S, Lakhal M, Fathallah C, Bouafsoun A, Kharrat M, Khemiri M et al. Molecular diagnosis of bacterial meningitis by multiplex real-time PCR in Tunisian children. J Infect Dev Ctries. 2018; 12:235-43. https://doi.org/10.3855/jidc. 9560

18. Brik A, Terrade A, Hong E, Deghmane A, Taha MK, Bouafsoun A et al. Phenotypic and genotypic characterization of meningococcal isolates in Tunis, Tunisia: High diversity and impact on vaccination strategies. Int J Infect Dis. 2020; 91:73-8. https://doi.org/10.1016/j. ijid.2019.11.013

19. Pai R, Gertz RE, Beall B. Sequential multiplex PCR approach for determining capsular serotypes of Streptococcus pneumoniae isolates. J Clin Microbiol. 2006;44(1):124-31. https://doi.org/10.1128/ JCM.44.1.124-131.2006

20. Nudelman Y, Tunkel AR. Bacterial menin- gitis: epidemiology, pathogenesis and management update. Drugs. 2009;69(18):2577-96. https://doi. org/10.2165/11530590-000000000-00000

21. Bai X, Hu X, Yan Q, Liu T, Qu B, Jiang N et al. Effects of meteorological factors on the incidence of meningococcal meningitis. Afri Health Sci. 2017;17(3):820-6. https://doi.org/10.4314/ahs. v17i3.25

22. National Institute of Statistics of Tunisia. Total Population by Governorates: Area and Gender Classification. Available at <http://regions.ins.tn/zbffpn/total-population-by-governorate-area-and-gender-classification?location $=$ Tunisia\&area-classification $=$ Total $>$; 2015. Accessed $13^{\text {th }}$ July 2019.

23. O'Brien KL. Current Status of PCV Use and WHO Recommendations. Available at <https://www. who.int/immunization/sage/meetings/2017/october/01_17_October_2017_Presentation_01_OBrien_ SAGE_PCV.pdf $>$; 2017. Accessed $14^{\text {th }}$ November 2018.

24. Badawi M, El Kholy A, Sherif MM, Rahman EA, Ashour, Sherif H et al. Serotypes of Streptococcus pneumoniae in Egyptian children: are they covered by pneumococcal conjugate vaccines? Eur J Clin Microbiol Infect Dis. 2017 ;36(12) :2385-389. https://doi. org/10.1007/s10096-017-3071-z

25. Azzari C, Nieddu F, Moriondo M, Indolfi G, Canessa C, Ricci $\mathrm{S}$ et al. Underestimation of Invasive Meningococcal Disease in Italy. Emerg Infect Dis. 2013;22(3):469-75. https://doi.org/10.1080/21645515 .2015 .1102811

26. Braikat M, Barkia A, El Mdaghri N, Rainey JJ, Cohen AL, Teleb N. Vaccination with Haemophilus influenzae type $\mathrm{b}$ conjugate vaccine reduces bacterial meningitis in Morocco. Vaccine. 2012;30(15):2594-9. https://doi.org/10.1016/j.vaccine.2012.01.041

27. Collins S, Litt D, Almond R, Findlow J, Ramsay $\mathrm{M}$, Borrow $\mathrm{R}$ et al. Haemophilus influenzae type $\mathrm{b}$ (Hib) seroprevalence and current epidemiology in England and Wales. J Infect. 2017;76(4):335-41. https://doi. org/10.1016/j.jinf.2017.12.010

28. Smaoui H, Saguer A, Bouziri A, Fourati S, Chahed MK, Ben Jaballah $\mathrm{N}$ et al. Les infections invasives à Neisseria meningitidis chez l'enfant à Tunis : à propos de 79 cas. Arch Inst Pasteur Tunis. 2011; 88:1-4.

29. Azevedo LC, Toscano CM, Bierrenbach AL. Bacterial Meningitis in Brazil: Baseline epidemiologic assessment of the decade prior to the introduction of pneumococcal and meningococcal vaccines. PLoS One. 2013;8(6): e64524. https://doi.org/10.1371/journal. pone.0064524 
30. Levy C, Bingen E, Aujard Y, Boucherat M, Floret D, Gendrel D et al. Observatoire national des méningites bactériennes de l'enfant en France : résultats de 7 années d'étude. Arch Pediatr. 2008; 15:99-104. https://doi.org/10.1016/S0929-693X(08)75491-5

31. Agrawal S, Nadel S. Acute bacterial meningitis in infants and children epidemiology and management. Paediatr Drugs. 2011; 13(6): 385-400. https://doi. org/10.2165/11593340-000000000-00000

32. Kuhdari P, Stefanati A, Lupi S, Valente N, Gabutti G. Meningococcal B vaccination: real-world experience and future perspectives. Pathog Glob Health. 2016;110(4-5):148-56. https://doi.org/10.1080/20477 724.2016 .1195072

33. Mohammed I, Iliyasu G, Habib AG. Emergence and control of epidemic meningococcal meningitis in sub-Saharan Africa. Pathog Glob Health. 2017;111(1):1-6. https://doi.org/10.1080/20477724.2016.1274068

34. World Health Organization. Pneumococcal conjugate vaccine for childhood immunization WHO position paper. Wkly Epidemiol Rec. 2007; 82:93104. Available at <http://www.who.int/wer/2007/ wer8212/en/> Accessed 1st May 2018.
35. Torres A, Bonnani P, Hryniewicz W, Moutschen M, Reinert RR, Welte T. Pneumococcal vaccination: what have we learnt so far and what can we expect in the future? Eur J Clin Microbiol Infect Dis. $2015 ; 34$ :19-31. https://doi.org/10.1016/j.ijid.2015.09.019

36. Diawara I, Zerouali K, Katfy K, Zaki B, Belabbes $\mathrm{H}$, Najib J et al. Invasive pneumococcal disease among children younger than 5 years of age before and after introduction of pneumococcal conjugate vaccine in Casablanca, Morocco. Int J Infect Dis. 2015; 40:95-101. https://doi.org/10.1016/j.ijid.2015.09.019

37. Tin Htar M, Christopoulos D, Schmitt HJ. Pneumococcal serotype evolution in Western Europe. BMC Infect Dis. 2015; 15:419. https:/ / doi.org/10.1186/ s12879-015-1147-x

38. Sadeq H, Husain EH, Alkoot A, Atyani S, Al-Fraij A, Al-Daithan A et al. Childhood meningitis in Kuwait in the era of post pneumococcal conjugate vaccination: A multicentre study. I Infect Public Health. 2017;10(6):766-9 https://doi.org/10.1016/j. jiph.2016.11.009 